\title{
Karim-Aly S. Kassam: Biocultural Diversity and Indigenous Ways of Knowing: Human Ecology in the Arctic
}

\author{
Calgary, Alberta: University of Calgary Press 2009 ISBN 978-1-55238-253-0, Price \\ $\$ 34.95$ US (paper). 270 pages, index
}

\section{Hua Qin}

Published online: 30 December 2010

(C) Springer Science+Business Media, LLC 2010

Human ecology has been broadly defined as the interdisciplinary study of the complex and varied systems of interaction between humans and their environment (Editors, Human Ecology 1972). In Biocultural Diversity and Indigenous Ways of Knowing, Karim-Aly Kassam provides a meaningful conceptual framework of human ecology integrating biological and cultural diversity with specific reference to the Circumpolar Arctic. Building on years of community-based research carried out in the Arctic region, he makes fine theoretical and empirical contributions to the further development of human ecological science.

The contents of this book can be organized into two sections. The first part presents a reconceptualization of human ecological relations within the context of indigenous Arctic communities. Kassam first introduces the intellectual origins of human ecology and critically examines its principal propositions based on an overview of recent research on the relationship between biological and cultural diversity. For him the fundamental flaw of the overall human ecological approach is the divide between nature and culture. However, refreshingly Kassam does not seek to offer a grand theory for unifying diverse human ecological research into a single discipline. Rather, he uses human ecology as a conceptual and analytic lens to bridge the schism between man and nature in the context of the Arctic and sub-Arctic. The circumpolar north is perceived as a homeland rather than simply a frontier for exploiting valuable natural resources. As reflected in the title of this volume, there are two core elements in Kassam's human

\section{H. Qin $(\varangle)$}

Climate Science and Applications Program,

National Center for Atmospheric Research,

PO Box 3000,

Boulder, CO 80307, USA

e-mail: huaqin@ucar.edu ecological framework for the Arctic: biocultural diversity and indigenous ecological knowledge. Diversity is the basis of sensory perception that forms a context for understanding relationships among human beings and between human and non-human organisms. Indigenous people's knowledge of their local environment is embedded in the contextual relationship between cultural and biological diversity, and constitutes the essence of human ecology for the circumpolar area.

The second part of the book includes three case studies which illustrate the interconnections between biological and cultural diversity and the continuance of indigenous ecological knowledge in specific Arctic community contexts. The first study describes the unique culture of the Arctic Inuit community of Ulukhaktok in Canada and provides details of local subsistence hunting and gathering. While this chapter provides an excellent narrative of local dependence on marine and terrestrial wildlife and plants, a more thorough description and discussion of community socio-ecological organization (e.g., community sharing of the fruits of the harvest and collective natural resource use and management) would enhance our understanding of the interactions among culturally prescribed behaviors, social structure, and the natural environment at Ulukhaktok. The methodological quality of this case study could also be improved with more complete information on the designing, sampling, and implementation procedures of the interviews.

The second case study explores local perceptions and knowledge of climate change impacts in the Inupiat community of Wainwright, Alaska. It makes a strong case for integrating conventional scientific and indigenous ways of knowing in climate change research and practices. The author highlights the applicability of community participation and action research methodology in human ecological investigation. However, some important information on the 
research process is again missing here. The third case study continues the discussion of the relationship between alternative ways of knowing using various examples of mapping of indigenous use of natural resources in the Arctic. It adds depth to the comparison of Western scientific and indigenous ecological knowledge with an analysis of the power relations inherent in knowledge production.

Human ecology is interdisciplinary in nature. The terms interdisciplinary and transdisciplinary are often used interchangeably. However, these two research approaches actually have distinct attributes and functions. Interdisciplinary research involves researchers from different disciplines working jointly on common problems within a shared conceptual framework. In contrast, transdisciplinary research emphasizes both the close collaboration of scholars across multiple disciplines and the engagement with nonacademic stakeholders in identifying and solving real-world problems (Wickson et al. 2006). In a sense, the type of community participatory research undertaken in all these three case studies represents a transdisciplinary trend in human ecology to go beyond integrating different disciplines and to incorporate indigenous or local knowledge into the study of human-environment interactions.

In sum, this well-written book contributes to a new perspective of human ecology by framing it as a worldview emphasizing the unity of culture and nature and by disseminating the author's cumulative empirical research findings from the Arctic and sub-Arctic region. I would recommend this book to anyone with an interest in the future of humans and their environment. The combination of well-grounded theoretical conceptualization and informative case studies makes it a suitable introduction to Arctic human ecology for both upper level undergraduate and beginning graduate students. It is also a valuable resource for researchers with broad interests in indigenous ecological knowledge, community-based environmental conservation, and human dimensions of climate change, and would be especially useful to policy makers and practitioners dealing with indigenous resource management issues. Finally, it is my genuine hope that the lessons learned from this work will find their way back to members of the Arctic communities, because only then can the dynamic and iterative process of phronesis (practical wisdom) generation described in the book be achieved and continued.

\section{References}

Editors (1972). Introductory statement. Human Ecology 1(1): 1. Wickson, F., Carew, A. L., and Russell, A. W. (2006). Transdisciplinary research: characteristics, quandaries and quality. Futures 38 (9): 1046-1059. 